\title{
LA RELACIÓN ENTRE LEALTAD Y SATISFACCIÓN DE CLIENTES: EL APORTE DEL NEUROMARKETING AL DEBATE
}

\author{
VÍCTOR VALENCIA POZO \\ Departamento de Administración \\ Facultad de Administración y Economía, Universidad de Santiago de Chile \\ victor.valencia@usach.cl \\ GERARDO NICOLÁS CASTILLEJO \\ Facultad de Ciencias Económicas y Administrativas, Universidad de Valparaíso, Chile \\ gerardo.castillejo@uv.cl
}

\section{RESUMEN}

Dada la importancia que tiene la base de clientes para la rentabilidad de las organizaciones, son innumerables los ejemplos de empresas que intentan resguardarse en la cuota de mercado y en la lealtad de sus clientes como fuente de ingresos. Lo anterior, parece provenir, según una parte de los investigadores como el resultado de una elevada satisfacción en los clientes. Podría resultar difícil de refutar una aseveración tan obvia y contundente, pero hay otra parte de los investigadores que promulgan lo contrario, es decir, que la lealtad no necesariamente proviene de una elevada satisfacción. La presente investigación, de carácter teórico, persigue analizar y comprender la divergencia de corrientes que existen en torno a la relación entre los conceptos de satisfacción y lealtad, incluyendo además una visión más actualizada con los aportes que el neuromarketing ha realizado en torno a esta discusión.

PALABRAS CLAVES: CUOTA DE MERCADO, LEALTAD, FIDELIZACIÓN, SATISFACCIÓN, NEUROMARKETING, ACELERADOR EMOCIONAL.

\section{ABSTRACT}

Given the importance that has the clients' base for the profitability of the organizations, there are innumerable the examples of companies that try to protect itself in the market share and in the loyalty of its clients as source of income. According to the above, it seems to come, according to a part of the investigators as the result of a high satisfaction in the clients. It mightturn out to be difficult to refute such an obvious and forceful affirmation, but there is another part of the investigators who promulgate the opposite, that is to say, that the loyalty not necessarily comes from a high satisfaction. The present investigation, of theoretical character, chases to analyze and to understand the difference of currents that exist concerning the relation between the concepts of satisfaction and loyalty, including in addition a vision more updated with the contributions that the neuromarketing has realized concerning this discussion.

KEY WORDS: MARKET SHARE, LOYALTY, FIDELIZATION, SATISFACTION, NEUROMARKETING, EMOTIONAL ACCELERATOR.

\section{INTRODUCCIÓN}

Hoy en día existe un gran consenso entre los investigadores a la hora de considerar la base de clientes de cualquier empresa como el activo más valioso. De su capacidad para gestionarla correctamente, depende la rentabilidad de las organizaciones, es por ello que se les ha otorgado a los clientes el papel central que les corresponde.

Debido a los cambios que se han producido en el entorno socio-económico actual, caracterizado por un aumento de la intensidad competitiva, apertura de los mercados, arribo de competencia extranjera, mayor incertidumbre y crisis que afectan simultáneamente a distintas regiones del mundo, es cada vez mayor el número de empresas que intentan resguardarse en su cuota de 
mercado mediante formulación de estrategias de lealtad de sus clientes.

La lealtad de marca es considerada uno de los aspectos determinantes para explicar la forma en que el consumidor selecciona entre las distintas opciones de marca, por ello este concepto ha generado un gran interés entre académicos y profesionales del marketing. Hoy se observan organizaciones que poseen amplias bases de clientes leales con grandes cuotas de mercado y elevadas tasas de rendimiento sobre la inversión (Jensen \& Hansen, 2006).

Por otra parte, la especialización de los sistemas informáticos, el manejo de base de datos de clientes y transacciones y los estudios de marketing relacional, han contribuido en la profundización del concepto de lealtad del consumidor en la última década (Delgado, 2004). Sin embargo, y debido a que la lealtad de marca es un fenómeno complejo abordado desde distintas perspectivas, aun sigue siendo un concepto fértil de análisis, donde se avizora el desarrollo de nuevas orientaciones y perspectivas de investigación, especialmente en los conceptos que pueden definir la lealtad: experiencia, emocionalidad, personalidad de marca, entre otros (Keller \& Lehmann, 2006).

Del mismo modo, existe un reconocimiento explícito de que la lealtad se encuentra estrechamente vinculada a la rentabilidad de la empresa, puesto que los clientes leales se caracterizan por tener una menor predisposición a cambiar de marca (Berné, Mugica \& Yaque, 1996), difundir comentarios positivos sobre la marca en el mercado, incrementar su volumen de compra (Reichheld \& Passer, 1990), incrementar las ventas cruzadas, admitir un sobreprecio (Diana, 1999) y relativizar las ofertas de la competencia, permitiéndole a las empresas bajar sus costos de comunicación y disminuir los costos de mantención (Hill \& Jones, 1996).
El objetivo del presente trabajo es de carácter exploratorio, y persigue analizar y relacionar los conceptos de: satisfacción, y lealtad de acuerdo con las tendencias existentes y el aporte del Neuromarketing en esta discusión.

\section{Algunos conceptos preliminares}

Los primeros estudios de comportamiento sobre lealtad se iniciaron en los años 70 y la mayoría de los investigadores midieron lealtad de acuerdo con un patrón de repetición de compra (Oliver, 1997). Jones \& Sasser (1995) definen lealtad de cliente como la intención por parte del cliente de repetir la compra de un determinado producto o servicio en el futuro. De acuerdo con la postura de los autores, la lealtad de cliente distingue o es distinta en función de largo o corto plazo. La de largo plazo implica una compra con un periodo largo en la cual no es fácil que el cliente cambie su elección.

Oliver (1997) define la lealtad como: el compromiso hacia la elección de repetir la compra o ser cliente efectivo de un producto o servicio en el futuro, a pesar de las influencias externas y de los esfuerzos comerciales que influyan potencialmente hacia un comportamiento de cambio. Esta definición incluye los tres niveles de lealtad propuestos por el autor: lealtad cognitiva, es decir la valoración de los atributos de la marca y por consiguiente la elección de una alternativa por ser considerada superior a las demás; lealtad afectiva, como compromiso del consumidor que presenta una actitud positiva hacia la marca derivada de la satisfacción; y lealtad conativa, que se manifiesta con el comportamiento de compra repetida. Una empresa logra fidelizar a sus clientes en la medida en que es capaz de conseguir una alta tasa de retención (Huete, 2003). La retención se podría definir como la repetición de una compra durante un determinado periodo. Fidelizar es lograr altas tasas de clientes que repiten a gusto o tasas bajas de no repetidores. 
Las definición de lealtad, en definitiva recoge una actitud positiva hacia la marca y la promesa, por parte del consumidor, de la recompra del producto. Esta actitud e intención son la consecuencia de la satisfacción del consumidor con la compra y llevarán a que la empresa obtenga mayores niveles de rentabilidad (Berné, 1996).

Barroso y Martín (1999, p. 16), indican que:

La lealtad de los clientes implica dar un paso más al estado emocional que proporciona la satisfacción y significa, en líneas generales, cuatro aspectos esenciales: primero: que el cliente está satisfecho; segundo: que recibe valor; tercero: que repite compra, y cuarto: que recomienda la empresa a terceros.

De acuerdo con De Juan Vigaray (2005):

La lealtad de una persona a un establecimiento o una marca puede ser definida como la inclinación a comprar la misma marca o a frecuentar dicho establecimiento para satisfacer una misma necesidad. El proceso de formación de la fidelidad, comienza con el valor percibido por el cliente hacia un establecimiento o la marca, el que repercutirá sobre su satisfacción y si esta es alta se conseguirá finalmente el objetivo de fidelización.

Por su parte, Dick y Basu (1994) definen la lealtad del cliente como la relación entre la actitud relativa al patrón de compra repetido.

\section{Importancia desde la perspectiva del marketing}

De acuerdo con el modelo de Reichheld (1996), el ciclo de crecimiento a base de lealtad, las fuerzas que gobiernan las relaciones recíprocas y los estados energéticos de las partículas elementales de un sistema de negocios (sus clientes, empleados e inversionistas) son las fuerzas de la lealtad. Por los vínculos que existen entre lealtad, valor y utilidades, estas fuerzas son mensurales en función del flujo de fondos. La lealtad está inextricablemente vinculada a la creación de valor como causa y como efecto. Como efecto, la lealtad mide en forma confiable si la compañía ha suministrado valor superior: los clientes, o bien vuelven por más, o bien se van a otra parte. Como causa, la lealtad inicia una serie de efectos económicos que se propagan en cascada a través del sistema del negocio, como sigue:

1. Los ingresos y la participación de mercado crecen cuando los mejores clientes son atraídos al negocio de la compañía, y se crean así ventas de repetición y remisiones. Como la oferta del valor de la compañía es fuerte, ella puede permitirse ser más selectiva en la adquisición de nuevos clientes y concentrar sus inversiones en los más rentables y potencialmente leales, estimulando así todavía más el crecimiento sostenible.

2. El crecimiento sostenible le permitirá a la firma atraer y conservar a los mejores empleados. El suministro constante de un valor superior a los clientes aumenta la lealtad de los empleados, pues para ellos es fuente de orgullo y satisfacción en el trabajo. Además los empleados antiguos aprenden a conocer a los viejos clientes y a suministrarles más valor aún, lo cual refuerza todavía más la lealtad del cliente y del empleado.

3. Los empleados antiguos y leales aprenden en el oficio cómo reducir costos y mejorar la calidad, lo que fortalece todavía más la oferta de valor al cliente y genera superior productividad. La compañía puede entonces aplicar ese superávit de productividad a financiar una superior remuneración y mejores herramientas de capacitación, lo cual refuerza más la productividad del empleado, su remuneración y su lealtad.

La espiral de productividad unida al aumento de eficiencia que proviene de tratar con clientes leales genera una ventaja de costos que a 
los competidores les es muy difícil de igualar. Una ventaja en costos sostenida, unida a un crecimiento continuo del número de clientes leales, genera utilidades que son muy atractivas para los inversionistas, lo cual le facilita a la firma atraer y conservar a los mejores inversionistas.

Los inversionistas leales se comportan como socios. Estabilizan el sistema, rebajan el costo de capital y aseguran que se vuelva a invertir en el negocio el capital necesario para financiar inversiones que aumente el potencial de creación de valor de la compañía.

La lealtad contribuye a proteger la vulnerabilidad de los resultados de la empresa elevando de manera sustancial las barreras de entrada a la competencia (Porter, 1982). Además se ha observado que con cada año adicional de relación entre una empresa y sus consumidores, se hace menos costoso atender a dicha clientela dado los efectos de aprendizaje que tienen lugar (Reichheld, 1993), es por ello, que la lealtad de marca es considerada como uno de los factores elementales del valor de una marca (Aaker, 1996).

\section{Lealtad, fidelidad ¿de qué depende?}

Tal como se comentó, los beneficios que conlleva la lealtad son numerosos y sería conveniente poder establecer cuáles son los factores que inducen a los clientes a mantener una relación a largo plazo con una compañía. En este sentido, existen trabajos que señalan que los clientes que perciben un mayor nivel de satisfacción presentan una mayor predisposición a mantener en el tiempo la relación y una mayor resistencia al cambio de proveedor (Fornell, 1992) frente a los clientes menos satisfechos.

La revisión de la literatura relacionada con el concepto de la satisfacción del consumidor conduce a los siguientes conceptos: Para Kotler \& Armstrong (2003) la satisfacción es la sensación que una persona experimenta de placer o decepción como consecuencia de comparar la percepción del desempeño de un producto en relación con sus expectativas. Si el desempeño es inferior a las expectativas, el cliente no estará satisfecho. Si el desempeño iguala las expectativas, el cliente estará satisfecho. Si el desempeño excede las expectativas, el cliente estará altamente satisfecho.

Por otra parte, Claver, Llopis \& Tari (1999) expresan que la satisfacción del cliente dependerá de la diferencia entre calidad percibida y la experimentada del producto o servicio; es decir, si la calidad percibida es mayor o igual a la esperada el cliente estará satisfecho, por el contrario, si la calidad percibida es menor a la esperada el cliente quedará insatisfecho. Para Vanhamme (2000) la satisfacción es un estado psicológico relativo que resulta de una experiencia de compra-consumo.

La satisfacción de clientes es un elemento central en la estrategia de las empresas. Se advierte en distintas corrientes de gestión, la inclusión de este aspecto como algo saludable en la estrategia de cualquier empresa. Es así, que el Balbrige Nacional Quality Program, en Estados Unidos, que se basa en la evaluación de siete categorías de criterios de desempeño, incluye la relación y nivel de satisfacción del cliente como una de ellas. Asimismo, la recogida y análisis de los datos relativos a la satisfacción del cliente es un requisito de la Norma ISO 9001:2000, e inclusive Hill \& Jones (1996) incluyen la superior capacidad de satisfacción del cliente como uno de los cuatro factores que constituyen la ventaja competitiva. Adicionalmente, existen herramientas de gestión como el Costumer Relationship Management (CMR) y el Balance Scorecard (Kaplan \& Norton, 1996) que incorporan indicadores de satisfacción del cliente como augurios de saludables resultados financieros.

Según un estudio realizado en una muestra de diferentes industrias (servicio de reparación de coches, depósitos bancarios, distribuidora industrial, lavandería industrial, construcción, soft- 
ware) por Reichheld y Sasser (1990): la reducción de un $5 \%$ en la tasa de abandono de los clientes puede aumentar los beneficios entre un 25\% y un $85 \%$, puesto que los clientes leales usan más los productos y servicios de la compañía adquiriendo mayores volúmenes de estos, a la vez que están dispuestos a pagar un mayor precio por ellos. Sin embargo, la afirmación de Reichheld (1996) no menciona que el costo de mantención y retención debe ser más bajo que los ingresos que genera dicho cliente. Más aún, dicha regla no puede ser aplicable a todos los clientes por igual, debido a que cada cliente genera una rentabilidad sensible, y en muchos casos, sustancialmente distinta. Si se sigue la idea de Kotler (2006), que dice que "muchas empresas miden la satisfacción del cliente, pero la gran mayoría no mide su rentabilidad individual" (p. 155), sería conveniente mencionar en la afirmación de Reichheld (1996), que los beneficios de la supuesta lealtad, estarían condicionados al tipo de industria y a la categorización de los clientes en términos de rentabilidad que haga la empresa.

Existen investigadores que sostienen que una elevada satisfacción provoca una elevada lealtad y que por ende, se produce un impacto favorable en la rentabilidad de las empresas (Berné, 1996), los clientes que están satisfechos comprarán de nuevo el mismo producto (Reichheld, 1996) y lo recomendarán a otros (Oliver \& Swan, 1989) Pero, en esta relación, no se ha explicitado qué sucede cuando las empresas se enfrentan a clientes muy sensibles al precio, y más aún, cuando enfrentan bajos costos de cambio, o son altamente sensibles a las acciones promocionales de la competencia.

Según Hallowell (1996), el fracaso de muchos programas de lealtad se debería a la premisa equivocada según la cual existe una relación directa entre la satisfacción y la lealtad del cliente, o entre la mayor satisfacción del cliente y la repetición de compra. Por el contrario, la repetición de compra no parece indicar que el cliente es leal, ya que la repetición puede producirse por otros motivos que no significan lealtad, tales como ausencia de alternativas en el mercado, guerra de precios, promociones efectivas (Jacoby \& Chesnut, 1973).

La satisfacción no es más que una actitud y no siempre existe correlación entre actitud y comportamiento (Cisneros \& Molina, 1996), por lo que una empresa puede perder clientes que están satisfechos, quienes simplemente se cambian a un competidor porque este es más competitivo en precios o bien como respuesta a un incentivo promocional. Por su lado Arrondo, Berné, Mugica \& Rivera (2002) señalan que, si bien la satisfacción podría ser considerada un requisito previo para la lealtad, no garantiza el compromiso de los clientes, sino que es necesario contar con respuestas afectivas.

Asimismo, Heskett (2003) estableció un modelo llamado "jerarquía de comportamiento del consumidor" en el que establece la relación que existe entre satisfacción y lealtad de los clientes. En dicho modelo se observa que no existe necesariamente una relación positiva entre satisfacción y lealtad, así, un cliente puede experimentar un alto grado de lealtad, y sentir un bajo nivel de satisfacción, tal como sería el caso de los clientes rehenes quienes poseen elevadas barreras de salida o enfrentan elevados costos de cambio.

Varios investigadores han argumentado acerca de los beneficios de tener clientes leales dentro de la cartera, diciendo que operan como los principales promotores de las compañías a través del boca en boca, que son aquellos que están dispuestos a pagar más por los productos que la empresa ofrece, que prestan menos atención a los mensajes publicitarios de la competencia, que toleran mejor una subida de precios y que cuesta menos servir. Algunas de estas aparentes ventajas producidas por la lealtad han sido rebatidas por algunos investigadores en los últimos años. Según Reinartz \& Kumar (2002) existe 
poca o ninguna evidencia que sugiera que los clientes que compran permanentemente a una empresa a lo largo del tiempo, sean menos costosos de servir, sean menos sensibles al precio o particularmente efectivos a la hora de traer nuevos clientes. En muchos casos, la satisfacción de clientes puede obtenerse a un costo elevado, e incluso superar la utilidad marginal entregada por ellos.

Finalmente, la cadena más grande de convenience-store de New England, llamada Store 24, decidió llevar adelante un programa para aumentar la satisfacción de sus clientes consistente en crear una atmósfera entretenida. Luego de dos años el programa debió ser abandonado por no mostrar resultados positivos, e incluso en muchos de sus locales se registraron pérdidas de clientes (Campbell, 2002).

Si bien existen autores que son defensores a ultranza de la relación directa que existe entre satisfacción y lealtad, y entre esta última y la rentabilidad, hay quienes promulgan lo contrario, generando zonas en el conocimiento que vale la pena indagar, investigar y analizar.

\section{EL APORTE DEL NEUROMARKETING AL DEBATE}

Según Joseph Leroux (2001), todas las capacidades mentales como la memoria, la percepción, imaginación, lenguaje y pensamiento tienen una compleja estructura subyacente. Las neurociencias cognitivas mejoran nuestra comprensión de estas capacidades, delineando los procesos componentes y especificando la forma en que estos interactúan.

\section{Neuromarketing}

El Neuromarketing (Felix, 2008) se entiende como el uso de los conocimientos variopintos de la investigación del cerebro por el marketing. Aunque el uso de los aparatos descritos más arri- ba desempeña un papel, la importancia considerablemente más grande es el hecho que este intenta integrar los descubrimientos completos de la investigación del cerebro de los últimos años en la teoría y la práctica del marketing. Es decir, que la investigación del cerebro ha descubierto muchos de los secretos de este en los últimos años, los cuales son de una importancia grande para el marketing. A continuación, algunas investigaciones relacionadas con la investigación.

- La investigación neurocientífica de la conciencia: la supremacía de los procesos inconscientes de la toma de decisiones.

- Los procesos multisensoriales de procesamiento en el cerebro.

- El estudio montague.

- Los procesos emocional-cognitivos de procesamiento de anuncios y tv-spots en el cerebro.

- La neurolingüística: el procesamiento de la lengua en el cerebro.

- La investigación neurocientífica de la personalidad.

\section{Limitaciones del neuromarketing}

"El neuromarketing está en su primera infancia y como tal está repleto de oportunidades de investigación, como se podría esperar, el paradigma es algo teórico y como tal, consiste en gran parte de estudios empíricos desunidos" (Garcia y Saas, p. 397)

La mayoría de los papers no son evolutivos y de base. La investigación evolutiva está basada en el método científico y menos sobre la colección de datos causales. Otro elemento importante es la contextualización Por ejemplo, en la utilización neuroimagingK, la tecnología para estudiar las políticas publicitarias y afiliaciones del consu- 
midor, es improbable que uno destape centros "conservadores" "o liberales" en el cerebro.

Sin embargo, la investigación puede ser dirigida en la búsqueda de regiones asociadas con el estado de reconocimiento y la toma de decisiones.

Cualquier nuevo campo de estudio, como el neuromarketing, necesita de la investigación revisada para establecer su importancia académica. El peligro de esta etapa en el ciclo de vida de un nuevo campo de estudio, consiste en que el foco puede estar en la dirección de las necesidades del especialista sin considerar un entorno más amplio.

\section{La satisfacción y la fidelidad parámetros poco estables según el neuromarketing}

Según explica Norbert Wittman (2009), la mayor parte de los anunciantes miden la satisfacción y algunos la fidelidad de los clientes, que son parámetros poco estables y que cambian muy rápido. Pero muy pocos hacen caso al valor de la confianza de los clientes.

La confianza es prácticamente la base de todas las decisiones de compra que se toman en el punto de venta, y esto quedó demostrado en el estudio realizado por Ebeltoft (2009) -consultora especializada en retail-. El estudio realizado a 10.000 personas se llamó Trust Research 2009, y analizó la confianza como factor decisivo para el éxito en 200 cadenas de establecimientos comerciales establecidas en once países de Europa.

Dentro de ese estudio se llegó a la conclusión de que los grandes almacenes son los establecimientos en los que más se confía, mientras que las tiendas de alimentación las resultaron las menos confiables.

\section{¿Cuáles son los indicadores de confianza, según los consumidores entonces?}

Dentro de ellos se destacan: Los buenos precios, que sea un negocio competente y bien organizado, que cuente con empleados profesionales y bien cualificados, que casi nunca decepcione, que sea honesto y cumpla siempre sus promesas, y que, en caso de no tenerlo cerca, se eche de menos.

Estos diversos testimonios se han concentrado en tres grandes conceptos, que se sitúan en los distintos ejes del modelo límbico del neuromarketing: por un lado, la competencia, situada entre los ejes de dominancia y equilibrio; los mismos ejes para el caso de la integridad, y por otra parte, la simpatía, entre el estímulo y el equilibrio.

Norbert Wittman (2009) explica que la confianza hace más sencilla nuestra vida: nos proporciona seguridad, social y general, reduce la complejidad y alienta las expectativas de recompensa. Además, señala que la peor consecuencia de la pérdida de confianza es la pérdida del cliente y destaca algunos de los hallazgos sobre la confianza como emotional booster, o 'acelerador emocional', en comercios y puntos de venta, obtenidos mediante estudios de neuromarketing.

Este mismo estudio señala que el principal motivo por el que un cliente deja de visitar un establecimiento es la antipatía del personal, motivo señalado como decisivo por el 36\% de los encuestados por Gruppe Nymphenburg. Le siguen la falta de limpieza, la falta de calidad, los precios demasiado elevados, la falta de visión de conjunto, la escasa selección de productos, la lentitud en caja y la ausencia de ambiente y de presencia. También se señalan algunos otros detalles relacionados con el ambiente del retail como la iluminación y la presentación de los productos influyen en la confianza de los clientes, como no exagerar con excesivas medidas de seguridad que muestren desconfianza hacia los propios 
clientes. Wittman remarca que cuando hay confianza en un establecimiento "casi nadie mira los precios".

Para este especialista, Christian Elger (2009), reputado epileptólogo y neurocientífico, la aplicación de la neurociencia a la neuroeconomía y el neuromarketing está generando una gran acumulación de conocimientos. Sin embargo, esta enorme fuente de conocimientos también es muy difícil de interpretar.

Las técnicas que se emplean para investigar el cerebro son la tomografía o imagen por resonancia magnética (IRM o TRM), que permite observar en imágenes la estructura y composición del cerebro, y la imagen por resonancia magnética funcional (IRMf), que permite ver en imágenes las áreas del cerebro que se activan al realizar determinadas acciones. El mayor problema con el que se encuentran las técnicas de investigación en neurociencia mediante resonancia magnética es en la medida de la diferencia. Las técnicas son capaces de reconocer las reacciones del cerebro, pero no se pueden establecer medidas. En todo caso se puede comparar una actividad cerebral con otra.

De acuerdo con Hausel y Hans (2009), nuestras decisiones de compra siempre se toman con el telón de fondo de nuestro sistema emocional. Para apelar a este sistema se pueden emplear múltiples mensajes: el embalaje, el olor, el sonido, la luz. Pero lo más sorprendente de todo es que la credibilidad de la escenificación de marketing no es decisiva: solo tiene que sonar creíble. Sin embargo, para el especialista Scheier (2001) esto no es cierto, las emociones están actualmente sobrevaloradas en publicidad. Los directivos de marketing tienen que preguntarse más bien si su marca apela al sistema de recompensas del cerebro. Cuando una persona no compra una marca, no es porque no entienda su mensaje o porque no crea en él, sino porque el significado de la marca no le ofrece suficientes recompensas.
Según el neurólogo Spitzer (2002) la secuencia de compra es la siguiente: las personas se motivan cuando consideran que algo es bueno, y lo consideran bueno porque reciben o recibirán una recompensa. Si nuestro cerebro decide que la "recompensa" nos satisface, se cierra el ciclo de la compra hacia la marca.

Si no hay recompensa, no hay acción, afirma Scheier (2001|). Esto demuestra en su opinión qué poco tiene que ver el ser humano con un ser movido por el instinto y las emociones. Entre la emoción y la acción se interpone el sistema de recompensas. Así, el valor de recompensa de la marca resulta decisivo.

\section{CONCLUSIONES}

Habría que destacar que cuando se habla de lealtad, se incluye tres niveles: lealtad cognitiva, es decir la valoración de los atributos de la marca y por consiguiente la elección de una alternativa por ser considerada superior a las demás; lealtad afectiva, como compromiso del consumidor que presenta una actitud positiva hacia la marca derivada de la satisfacción; y lealtad conativa, que se manifiesta con el comportamiento de compra repetida.

Para muchos autores el grado de satisfacción experimentado por los consumidores con el producto / servicio guarda relación directa con la lealtad obtenida. Cabe precisar, que esta referencia es con respecto a lo que se conoce como lealtad afectiva.

La lealtad está inextricablemente vinculada a la creación de valor como causa y como efecto. Como efecto, la lealtad mide en forma confiable si la compañía ha suministrado valor superior a los clientes, o bien vuelven por más, o bien se van a otra parte. Como causa, la lealtad inicia una serie de efectos económicos positivos para la empresa que se propagan en cascada a través del sistema del negocio. 
Existen investigadores que sostienen que una elevada satisfacción provoca una elevada lealtad y que por ende, se produce un impacto favorable en la rentabilidad de las empresas. Sin embargo, en esta relación no se ha explicitado que sucede cuando las empresas se enfrentan a clientes muy sensibles al precio, y más aún, cuando enfrentan bajos costos de cambio o son altamente sensibles a las acciones promocionales de la competencia.

Para otro gran grupo de autores, es una premisa equivocada la existencia de una relación directa entre la satisfacción y la lealtad del cliente, o entre la mayor satisfacción del cliente y la repetición de compra. Por el contrario, la repetición de compra no parece indicar que el cliente es leal, ya que la repetición puede producirse por otros motivos que no significan lealtad, tales como ausencia de alternativas en el mercado, guerra de precios o promociones efectivas.

Del neuromarketing emergen otro concepto que dice relación con la lealtad, esta es la confianza. La confianza es prácticamente la base de todas las decisiones de compra que se toman en el punto de venta o dicho de otro modo de la lealtad conativa.

Estudios de neuromarketing señalan que la peor consecuencia de la pérdida de confianza es la pérdida del cliente y que esta actúa como emotional booster, o 'acelerador emocional', en comercios y puntos de venta.

Cuando una persona no compra una marca, no es porque no entienda su mensaje o porque no crea en él, sino porque el significado de la marca no le ofrece suficientes recompensas (confianza). Si nuestro cerebro decide que la "recompensa" nos satisface, se cierra el ciclo de la compra hacia la marca.

En opinión de algunos esto demuestra qué poco tiene que ver el ser humano con un ser movido por el instinto y las emociones. Entre la emoción y la acción se interpone el sistema de recompensas. Así, el valor de recompensa de la marca resulta decisivo.

En definitiva, y de acuerdo con lo examinado, no existen verdades universales que hagan suponer relaciones positivas entre satisfacción y lealtad. Bajo ciertas condiciones parece darse la relación positiva esperada, pero bajo otras circunstancias la relación es fuertemente cuestionada. El neuromarketing aporta nuevos conceptos en torno a la discusión, ya que incluye otros aspectos como el "sistema de recompensas" que resulta decisivo para determinar la compra y repetición de la compra por parte del consumidor promedio.

\section{REFERENCIAS}

Aaker, D. (1996). Construir Marcas Poderosas. Madrid: Ediciones Gestión 2000 S.A.

Arrondo, E., Berné, C., Mugica, J.M. \& Rivera, P. (2002). ModeIling Of Customer Retention In Multi-Format Retailing. International Review Of Retail, Distribution And Consumer Research, 12(3), 281-296.

Barroso, C. \& Martín, E. (1999). Marketing Relacional. Madrid: Esic.

Berné, C., Mugica, J.M. \& Yaque, M.J. (1996). La Gestión Estratégica y Los Conceptos De Calidad Percibida, Satisfacción Del Cliente y Lealtad. Economía Industrial, 307, 63-74.

Cammbell, D. (2002).Putting Strategy Hypotheses To The Test With Cause-And-Effect Analysis. Balanced Scorecard Report, September-October, 3.

Cisneros, G. \& Molina, J. (1996). Fidelización Efectiva: No Caiga En Los Errores Más Frecuentes. Harvard Deusto Marketing y Ventas, Noviembre-Diciembre, 30-35.

Claver, E., Llopis, J. \& Tarí, J. J. (1999). Calidad y Dirección De Empresas. Madrid: Civitas

De Juan Vigaray, M. D. (2005). Comercialización y Retailing. Madrid: Pearson Prentice Hall.

Delgado, E. (2004). Estado Actual De La Investigación Sobre Lealtad De Marca: Una Revisión Teórica. Revista De Dirección, Organización y Administración De Empresas, 30, 16-24. 
Diana, F. (1999). Fidelización De Clientes y Rentabilidad. Coev Economistes, 162 (2a Quincena De Mayo).

Dick, A. \& Basu, K. (1994). Customer Loyalty: Toward And Integrated Conceptual Framework. Journal OfDe Academy OfMarketing Science, 22( 2), 89-113.

Fornell, C. (1992). A National Customer Satisfaction Barometer: The Swedish Experience. Journal Of Marketing, 56, 6-21.

Hallowell, R. (1996). The Relationships Of Customer Satisfaction, Customer Loyalty, And Profitability: An Empirical Study. International Journal Of Service Industry Management, 7, 27-42.

Heskett, J. L., Sasser W. Jr. \& Schlesinger, L. (2003). The Value Profit Chain: Treat Employees Like Customers And Customers Like Employees. N.Y.: The Free Press

Hill, C. W., Jones, L. \& Gareth, R. (1996). Administración Estratégica, Un Enfoque Integrado. Santa Fé De Bogotá: Mc Graw Hill.

Hubert, M. \& Kenning, P. (2008). A Current Overview Of Consumer Neurosciecie, Journal Of Consumer Behaviour July-October.

Huete, L. (2003). Servicios \& Beneficios. Barcelona: Ediciones Deusto

Huxley, A. (1932). Brave New World. Londres: Chatto And Windus,

Jacobi, J. \& Chestnut, R, (1973). Brand Loyalty Vs. Repeat Purchasing Behavior. Journal Of Marketing Research, 10, 1-9.

Jensen, J. \& Hansen, T. (2006). An Empirical Examination Of Brand Loyalty. Journal Of Product \& Brand Management, 15(7), 442-449.

Jones, T. O. \& Sasser, W. E. (1995). Why Satisfied Customers Effect. Harvard Business Review. November-December, 88-99

Justin, R Garcia \& Gad Saad (2008). Evolutionary Neuromarketing: Darwinizing The Neuroimaging Paradigm For Consumer Behavior By, . London: Jul-Oct 2008. Vol. 7, Iss. 4/5; P. 397
Kaplan R. S. \& Norton David P. (1996). Cuadro De Mando Integral (The Balanced Scorecard). Barcelona: Gestión 2000.

Keller, K.L. \& Lehmann, D. (2006) Brands And Branding: Research Findings And Future Priorities. Marketing Science, 25(5), 740-759.

Kotler, P. (2006). Cómo Crear, Ganary Dominar Mercados. España: Paidós Ibérica.

Kotler, P. \& Armstrong, G. (2003). Fundamentos De Marketing. México: Pearson Prentice Hall.

Oliver, R. L. (1999). Whence Consumer Loyalty. Journal Of Marketing, 63, 33-44.

Oliver, R. L. \& Swan, J. D. (1989). Consumer Perceptions Of Interpersonal Equity And Satisfaction In Transactions: A Field Survey Approach. Journal Of Marketing, 53, 2135.

Porter, M. E. (1997). Estrategia Competitiva. México: Cecsa.

Reichheld, F. F. (1996). El Efecto De La Lealtad, La Fuerza Oculta Detrás Del Crecimiento, La Rentabilidad y La Creación De Valor En Las Empresas. Bogotá: Norma.

Reichheld, F.F. (1993). Loyalty-Based Management. Harvard Business Review, Marzo-Abril, 64-73.

Reichheld, F.F. \& Sasser, W. (1990). Zero Defections: Quality Comes To Services. Harvard Business Review, Sept-Oct, 105-111.

Rienartz, W. \& Kuman, V. (2002). The Mismanagement Of Costumer Loyalty. Harvard Business Review, Julio, 4-12.

Scheier, C. (2001): Understanding The Intelligence, September, The Mit Press.

Spitzer, M. (2002). Exploratory Analysis And Data Modeling In Funcional Neuroimaging, December 2002, The Mit Press.

Vanhamme, J. (2000). The Link Between Surprise And Satisfaction: An Exploratory Research. Journal Of Marketing Management, 16(6), 565-582.

Recibido: 16-06-2011

Aceptado: 08-11-2011 